\title{
EXTENSION OF AN INEQUALITY WITH POWER EXPONENTIAL FUNCTIONS
}

\author{
MITSUHIRO MIYAGI AND YUSUKE NISHIZAWA
}

\begin{abstract}
V. Cîrtoaje et al. [2] conjectured and proved [3, 4] that the inequality $a^{r b}+$ $b^{r a} \leq 2$ holds for all nonnegative numbers $r \leq 3$ and nonnegative real numbers $a, b$ with $a+b=2$. In this paper, we will show that $a^{r b}+b^{r a} \leq 2$ holds for all nonnegative $r \geq 3$ and all nonnegative real numbers $a, b$ with $a+b=2$ and some conditions. This gives an extended inequality of conjectured by V. Cîrtoaje.
\end{abstract}

\section{Introduction}

Inequalities appear on the various branches of mathematics. In this paper, we give a result of an inequality with power exponential functions which is studied by V. Cîrtoaje et al. $[1,2,3,4,5,6,7,8]$. The formula of inequalities with power exponential functions are very simple, but their proof is not as simple as it seems. V. Cîrtoaje et al. [3,4] proved that the inequality

$$
a^{r b}+b^{r a} \leq 2
$$

holds for all nonnegative real number $r \leq 3$ and all nonnegative real numbers $a, b$ with $a+b=$ 2. Miyagi et al. [7] proved that the stronger inequality

$$
a^{3 b}+b^{3 a}+\left(\frac{a-b}{2}\right)^{4} \leq 2
$$

holds for the same conditions. These inequalities (1.1) and (1.2) are conjectures by V. Cîrtoaje [2]. The following is our main theorem.

Theorem 1.1. The inequality

$$
a^{r b}+b^{r a} \leq 2
$$

holds for all numbers $r \geq 3$ and all real numbers $a, b \in\left[0,1-((r-3) /(r-2))^{1 / 3}\right] \cup[1+((r-$ 3) $\left./(r-2))^{1 / 3}, 2\right]$ with $a+b=2$.

Received January 15, 2015, accepted March 15, 2015. 2010 Mathematics Subject Classification. Primary 26D10.

Key words and phrases. Power-exponential function, monotonically decreasing function, monotonically increasing function.

Corresponding author: Yusuke Nishizawa. 
The above inequality (1.3) is an extension of the inequality (1.1).

\section{Preliminaries}

In this section, we will show some lemmas to prove our main theorem.

Lemma 2.1. If $0<b<1$, then

$$
(2-b)^{3 b-1} \ln (2-b)+b^{5-3 b} \ln b>0 .
$$

\section{Proof.}

$$
(2-b)^{3 b-1} \ln (2-b)+b^{5-3 b} \ln b>0
$$

is equivalent to

$$
\frac{-(b(2-b))^{3 b} \ln (2-b)}{(2-b) b^{5} \ln b}>1 .
$$

We set

$$
f(b)=b^{3 b-4}-(2-b)
$$

then we have derivatives

$$
f^{\prime}(b)=1+b^{3 b-4}\left(\frac{3 b-4}{b}+3 \ln b\right)
$$

and

$$
f^{\prime \prime}(b)=b^{3 b-4}\left(\frac{3}{b}+\frac{4}{b^{2}}\right)+b^{3 b-4}\left(\frac{3 b-4}{b}+3 \ln b\right)^{2} .
$$

Since $f^{\prime \prime}(b)>0, f^{\prime}(b)$ is strictly increasing for $b$. Since $f^{\prime}(1)=0$, we have $f^{\prime}(b)<0$ for all $0<b<1$. Therefore, $f(b)$ is strictly decreasing for $0<b<1$. Since $f(1)=0$, we have $f(b)>0$ for all $0<b<1$. Therefore, we get

for all $0<b<1$. We set

$$
\frac{b^{3 b-4}}{2-b}>1
$$

$$
g(b)=\ln (2-b)+b \ln b
$$

then we have derivatives

and

$$
g^{\prime}(b)=1-\frac{1}{2-b}+\ln b
$$

$$
g^{\prime \prime}(b)=\frac{(b-4)(b-1)}{(b-2)^{2} b} .
$$

Since $g^{\prime \prime}(b)>0, g^{\prime}(b)$ is strictly increasing for $b$. Since $g^{\prime}(1)=0$, we have $g^{\prime}(b)<0$ for all $0<b<1$. Therefore, $g(b)$ is strictly decreasing for $0<b<1$. Since $g(1)=0$, we have $g(b)>0$ for all $0<b<1$. Therefore, we get

$$
-\frac{\ln (2-b)}{b \ln b}>1
$$


for all $0<b<1$. Since $2-b>1$ and $(2-b)^{3 b}>1^{3 b}=1$, we have $(2-b)^{3 b}>1$. Thus, we can get

$$
\frac{-(b(2-b))^{3 b} \ln (2-b)}{(2-b) b^{5} \ln b}>1 \text {. }
$$

for all $0 \leq b \leq 1$.

Lemma 2.2. If $0<t<1$, then $G_{1}(t)>0$, where

$$
G_{1}(t)=18+54 t+45 t^{2}+12 t^{3}-63 t^{4}-60 t^{5}-22 t^{6}+36 t^{7}+30 t^{8}+16 t^{9}>0 .
$$

Proof. We set

$$
f(t)=6+45 t^{2}+12 t^{3}-63 t^{4}
$$

and

$$
g(t)=12+54 t-60 t^{5}-22 t^{6}+36 t^{7} .
$$

Since $f^{\prime}(t)=18 t\left(5+2 t-14 t^{2}\right)$ and $f^{\prime}(0)=f^{\prime}((1+\sqrt{71}) / 14)=0, f(t)$ is strictly increasing for $0<t<(1+\sqrt{71}) / 14$ and $f(t)$ is strictly decreasing for $(1+\sqrt{71}) / 14<t<1$. From $f(0)=6$ and $f(1)=0, f(t)>0$ for all $0<t<1$. Since $g^{\prime}(t)=6\left(9-50 t^{4}-22 t^{5}+42 t^{6}\right)$ and $g^{\prime \prime}(t)=$ $12 t^{3}\left(-100-55 t+126 t^{2}\right)<0, g^{\prime}(t)$ is strictly decreasing for $0<t<1$. From $g^{\prime}(0)=54$ and $g^{\prime}(1)=-126$, there exists uniquely a number $t_{0}$ with $0<t_{0}<1$ such that $g^{\prime}\left(t_{0}\right)=0$. Since $g^{\prime}(t)>0$ for $0<t<t_{0}$ and $g^{\prime}(t)<0$ for $t_{0}<t<1, g(t)$ is strictly increasing for $0<t<t_{0}$ and $g(t)$ is strictly decreasing for $t_{0}<t<1$. From $g(0)=12$ and $g(1)=20, g(t)>0$ for all $0<t<1$. Since $G_{1}(t)>f(t)+g(t)$ and $f(t)+g(t)>0$, we have $G_{1}(t)>0$ for $0<t<1$.

Lemma 2.3. There exists uniquely a number $t_{1}$ with $0<t_{1}<1$ such that $G_{2}\left(t_{1}\right)=0, G_{2}(t)<0$ for $0<t<t_{1}$ and $G_{2}(t)>0$ for $t_{1}<t<1$, where

$$
G_{2}(t)=-18+18 t+9 t^{2}+36 t^{3}+24 t^{5}+2 t^{6}+16 t^{7} .
$$

Proof. From $G_{2}^{\prime}(t)>0, G_{2}(t)$ is strictly increasing for $0<t<1$. Since $G_{2}(0)=-18$ and $G_{2}(1)=$ 87, there exists uniquely a number $t_{1}$ with $0<t_{1}<1$ such that $G_{2}\left(t_{1}\right)=0$. Therefore, we have $G_{2}(t)<0$ for $0<t<t_{1}$ and $G_{2}(t)>0$ for $t_{1}<t<1$.

Lemma 2.4. If $0<t<1$, then

$$
H_{1}(t)>0, \quad H_{2}(t)>0, \quad H_{3}(t)>0,
$$

where

$$
\begin{aligned}
H_{1}(t)= & 648-5184 t+13986 t^{2} \\
H_{2}(t)= & 31320 t^{3}+73143 t^{4}+14742 t^{5}-35433 t^{6}-137844 t^{7} \\
& -53988 t^{8}-2000 t^{9}-3828 t^{9}+121410 t^{10} \\
H_{3}(t)= & 50100 t^{11}+44862 t^{12}-36280 t^{13}+7156 t^{14} \\
& -20384 t^{15}+1408 t^{16}-22064 t^{17}-840 t^{18}
\end{aligned}
$$


Proof. We have following inequalities

and

$$
H_{1}(t)>H_{1}\left(\frac{48}{259}\right)=\frac{43416}{259}
$$

$$
\begin{aligned}
H_{2}(t)> & 30000 t^{3}+70000 t^{4}+14000 t^{5}-36000 t^{6}-138000 t^{7} \\
& -54000 t^{8}-2000 t^{9}-4000 t^{9}+120000 t^{10} \\
= & 2000(-1+t) t^{3}\left(-15-50 t-57 t^{2}-39 t^{3}+30 t^{4}+57 t^{5}+60 t^{6}\right) .
\end{aligned}
$$

Here, we set

$$
\begin{aligned}
& f(t)=-39 t^{3}+30 t^{4} \\
& g(t)=-57 t^{2}+57 t^{5}
\end{aligned}
$$

and

$$
h(t)=-15-50 t+60 t^{6} .
$$

Then we have following inequalities

$$
\begin{aligned}
& f(t)=3 t^{3}(-13+10 t)<3 t^{3}(-13+10)=-9 t^{3}<0 \\
& g(t)=57(-1+t) t^{2}\left(1+t+t^{2}\right)<0
\end{aligned}
$$

and

$$
h(t)=-15+10 t\left(-5+6 t^{5}\right)<-15+10 t(-5+6)<-5 .
$$

Since $f(t)+g(t)+h(t)<0$, we have $H_{2}(t)>0$. We have

$$
\begin{aligned}
H_{3}(t) & >50000 t^{11}+44000 t^{12}-37000 t^{13}+7000 t^{14}-21000 t^{15}+1400 t^{16} \\
& -23000 t^{17}-1000 t^{18} \\
& =-200 t^{11}\left(-250-220 t+185 t^{2}-35 t^{3}+105 t^{4}-7 t^{5}+115 t^{6}+5 t^{7}\right) .
\end{aligned}
$$

Since

$$
-250+105 t^{4}+115 t^{6}+5 t^{7}<-250+105+115+5<0
$$

and

$$
\begin{aligned}
-220 t+185 t^{2}-35 t^{3}-7 t^{5} & =-t\left(220-185 t+35 t^{2}+7 t^{4}\right) \\
& <-t(220-185 t) \\
& <0
\end{aligned}
$$

we have $H_{3}(t)>0$. Therefore, we have $H_{1}(t)>0, H_{2}(t)>0$ and $H_{3}(t)>0$ for $0<t<1$.

Lemma 2.5. If $0 \leq t \leq 1$, then $G(t) \leq 0$, where

$$
G(t)=e^{(1+t)\left(\frac{1}{1-t^{3}}+2\right)\left(-t-\frac{t^{2}}{2}-\frac{t^{3}}{3}\right)}+e^{(1-t)\left(\frac{1}{1-t^{3}}+2\right)\left(t-\frac{t^{2}}{2}+\frac{t^{3}}{3}\right)}-2 .
$$


Proof. We have

$$
G^{\prime}(t)=e^{(1+t)\left(\frac{1}{1-t^{3}}+2\right)\left(-t-\frac{t^{2}}{2}-\frac{t^{3}}{3}\right)} \frac{-G_{1}(t)}{6(-1+t)^{2}\left(1+t+t^{2}\right)^{2}}+e^{(1-t)\left(\frac{1}{1-t^{3}}+2\right)\left(t-\frac{t^{2}}{2}+\frac{t^{3}}{3}\right)} \frac{-G_{2}(t)}{6\left(1+t+t^{2}\right)^{2}}
$$

where

$$
G_{1}(t)=18+54 t+45 t^{2}+12 t^{3}-63 t^{4}-60 t^{5}-22 t^{6}+36 t^{7}+30 t^{8}+16 t^{9}
$$

and

$$
G_{2}(t)=-18+18 t+9 t^{2}+36 t^{3}+24 t^{5}+2 t^{6}+16 t^{7} .
$$

According to Lemmas 2.2 and 2.3, we have $G_{1}(t)>0$ and $G_{2}(t) \geq 0$ for $t_{1} \leq t<1$, therefore $G^{\prime}(t)<0$ for $t_{1} \leq t<1$. We will show further that $G^{\prime}(t)$ is also negative for $0<t<t_{1}$, which involves $G^{\prime}(t)<0$ for $0<t<1$. The inequality $G^{\prime}(t)<0$ for $0<t<t_{1}$ is equivalent to $H(t)>0$, where

$$
\begin{aligned}
H(t)= & (1+t)\left(-t-\frac{t^{2}}{2}-\frac{t^{3}}{3}\right)\left(\frac{1}{1-t^{3}}+2\right)+\ln G_{1}(t)-\ln \left((-1+t)^{2}\right) \\
& -(1-t)\left(t-\frac{t^{2}}{2}+\frac{t^{3}}{3}\right)\left(\frac{1}{1-t^{3}}+2\right)-\ln \left(-G_{2}(t)\right)>0 .
\end{aligned}
$$

The derivative of $H(t)$ is

where

$$
H^{\prime}(t)=\frac{-t^{2}\left(H_{1}(t)+H_{2}(t)+H_{3}(t)\right)}{(-1+t)^{2}\left(1+t+t^{2}\right)^{2} G_{1}(t) G_{2}(t)},
$$

$$
\begin{gathered}
H_{1}(t)=648-5184 t+13986 t^{2} \\
H_{2}(t)=31320 t^{3}+73143 t^{4}+14742 t^{5}-35433 t^{6}-137844 t^{7} \\
-53988 t^{8}-2000 t^{9}-3828 t^{9}+121410 t^{10}
\end{gathered}
$$

and

$$
\begin{aligned}
H_{3}(t)= & 50100 t^{11}+44862 t^{12}-36280 t^{13}+7156 t^{14} \\
& -20384 t^{15}+1408 t^{16}-22064 t^{17}-840 t^{18} .
\end{aligned}
$$

By Lemma 2.4, it follows that $H^{\prime}(t)>0$ for $0<t<t_{1}$, when $G_{1}(t)>0$ and $G_{2}(t)<0$. Therefore, $H(t)$ is strictly increasing for $0<t<t_{1}$, hence $H(t)>H(0)=0$. Thus, $G^{\prime}(t)<0$ for $0<t<1$, $G(t)$ is strictly decreasing, $G(t)<G(0)=0$ for $t_{1}<t \leq 1$.

\section{Proof of Theorem 1.1}

Proof. Without loss of generically, we assume that

$$
0 \leq b \leq 1-\left(\frac{r-3}{r-2}\right)^{\frac{1}{3}}
$$


and

$$
1+\left(\frac{r-3}{r-2}\right)^{\frac{1}{3}} \leq a \leq 2
$$

We set

$$
F(b, r)=(2-b)^{r b}+b^{r(2-b)}-2 .
$$

Then we have derivatives

and

$$
\frac{\partial F}{\partial r}(b, r)=(2-b)^{b r} b \ln (2-b)+(2-b) b^{(2-b) r} \ln b
$$

$$
\frac{\partial^{2} F}{\partial r^{2}}(b, r)=(2-b)^{b r} b^{2}(\ln (2-b))^{2}+(2-b)^{2} b^{(2-b) r}(\ln b)^{2} .
$$

Since $\partial^{2} F(b, r) / \partial r^{2} \geq 0$, the function $\partial F(b, r) / \partial r$ is strictly increasing for $r$. By Lemma 2.1, we have

$$
\begin{aligned}
\frac{\partial F}{\partial r}(b, r) & \geq \frac{\partial F}{\partial r}(b, 3) \\
& =b(2-b)\left((2-b)^{3 b-1} \ln (2-b)+b^{5-3 b} \ln b\right) \\
& \geq 0 .
\end{aligned}
$$

Thus, $F(b, r)$ is strictly increasing for $r \geq 3$. Since

$$
0 \leq b \leq 1-\left(\frac{r-3}{r-2}\right)^{\frac{1}{3}}
$$

we have

$$
3 \leq r \leq \frac{1}{1-(1-b)^{3}}+2
$$

Thus, we can get

$$
\begin{aligned}
F(b, r) & \leq F\left(b, \frac{1}{1-(1-b)^{3}}+2\right) \\
& =(2-b)^{\left(\frac{1}{1-(1-b)^{3}}+2\right) b}+b^{\left(\frac{1}{1-(1-b)^{3}}+2\right)(2-b)}-2 .
\end{aligned}
$$

Therefore, it suffices to show that

$$
(2-b)^{\left(\frac{1}{1-(1-b)^{3}}+2\right) b}+b^{\left(\frac{1}{1-(1-b)^{3}}+2\right)(2-b)}-2 \leq 0 .
$$

Denoting

$$
t=1-b, \quad 0 \leq t \leq 1,
$$

this desired inequality becomes

$$
(1+t)^{(1-t)\left(\frac{1}{1-t^{3}}+2\right)}+(1-t)^{(1+t)\left(\frac{1}{1-t^{3}}+2\right)}-2 \leq 0 .
$$

From Lemma 6.1 in [3], we have

$$
\ln (1+t) \leq t-\frac{t^{2}}{2}+\frac{t^{3}}{3}
$$


for all $t>-1$. Using this inequality, we get

$$
\begin{aligned}
& (1-t)^{(1+t)\left(\frac{1}{1-t^{3}}+2\right)}+(1+t)^{(1-t)\left(\frac{1}{1-t^{3}}+2\right)}-2 \\
& \quad=e^{(1+t)\left(\frac{1}{1-t^{3}}+2\right) \ln (1-t)}+e^{(1-t)\left(\frac{1}{1-t^{3}}+2\right) \ln (1+t)}-2 \\
& \quad \leq e^{(1+t)\left(\frac{1}{1-t^{3}}+2\right)\left(-t-\frac{t^{2}}{2}-\frac{t^{3}}{3}\right)}+e^{(1-t)\left(\frac{1}{1-t^{3}}+2\right)\left(t-\frac{t^{2}}{2}+\frac{t^{3}}{3}\right)}-2 .
\end{aligned}
$$

Therefore, it suffices to prove that $G(t) \leq 0$ for $0 \leq t \leq 1$, where

$$
G(t)=e^{(1+t)\left(\frac{1}{1-t^{3}}+2\right)\left(-t-\frac{t^{2}}{2}-\frac{t^{3}}{3}\right)}+e^{(1-t)\left(\frac{1}{1-t^{3}}+2\right)\left(t-\frac{t^{2}}{2}+\frac{t^{3}}{3}\right)}-2 .
$$

This is true by Lemma 2.5. Thus, the proof of Theorem 1.1 is completed.

\section{Acknowledgements}

We would like to thank the referees for their valuable comments.

\section{References}

[1] A. Coronel and F. Huancas, On the inequality $a^{2 a}+b^{2 b}+c^{2 c} \geq a^{2 b}+b^{2 c}+c^{2 a}$, Aust. J. Math. Anal. Appl., 9 (2012), Art. 3.

[2] V. Cîrtoaje, On some inequalities with power-exponential functions, J. Ineq. Pure Appl. Math., 10 (2009), Art. 21.

[3] V. Cîrtoaje, Proofs of three open inequalities with power-exponential functions, J. Nonlinear Sci. Appl., 4 (2011), 130-137.

[4] L. Matejicka, Solution of one conjecture on inequalities with power-exponential functions, J. Ineq. Pure Appl. Math., 10 (2009), Art. 72.

[5] L. Matejicka, Proof of one open inequality, J. Nonlinear Sci. Appl., 7 (2014), 51-62.

[6] M. Miyagi and Y. Nishizawa, Proof of an open inequality with double power-exponential functions, J. Inequal. Appl. 2013, 2013:468.

[7] M. Miyagi and Y. Nishizawa, A short proof of an open inequality with power-exponential functions, Aust. J. Math. Anal. Appl., 11 (2014), Art. 6.

[8] M. Miyagi and Y. Nishizawa, A stronger inequality of Cîrtoaje's one with power exponential functions, J. Nonlinear Sci. Appl., 8 (2015), 224-230.

General Education, Ube National College of Technology, Tokiwadai 2-14-1, Ube, Yamaguchi 755-8555, Japan.

E-mail: miyagi@ube-k.ac.jp

General Education, Ube National College of Technology, Tokiwadai 2-14-1, Ube, Yamaguchi 755-8555, Japan.

E-mail: yusuke@ube-k.ac.jp 